\title{
Clinical Predictors of Postintubation Bilateral Vocal Fold Immobility
}

\author{
Erin Rachel Cohen ${ }^{1}$ Cortney Lee Dable ${ }^{10}$ Thomas Iglesias ${ }^{1(0)}$ Eshita Singh ${ }^{1}$ Ruixuan Ma ${ }^{2}$ \\ David Edward Rosow ${ }^{1}$ (i)
}

${ }^{1}$ Department of Otolaryngology, University of Miami Miller School of Medicine, Miami, Florida, United States

2 Division of Biostatistics, Department of Public Health Sciences, University of Miami Miller School of Medicine, Miami, Florida, United States

Int Arch Otorhinolaryngol 2022;26(4):e524-e532.
Address for correspondence David E. Rosow, MD, FACS, Department of Otolaryngology, University of Miami Miller School of Medicine, 1120 NW 14th Street, Miami, FL 33136-1015, United States (e-mail: drosow@med.miami.edu).

\begin{abstract}
Keywords

- vocal cord paralysis

- tracheostomy

- device removal

- laryngostenosis

- glottis

Introduction latrogenic bilateral vocal fold immobility (BVFI) often arises from posterior glottic stenosis (PGS) after endotracheal intubation, whereby posterior commissure mucosal disruption leads to fibrosis and ankylosis of the cricoarytenoid joints. Sequelae can be devastating, resulting in dyspnea, stridor, and death due to asphyxiation.

Objectives We sought to review features associated with PGS to better understand how to prevent this condition. A secondary aim is to analyze factors correlating to tracheostomy dependence.

Methods Charts from January 2010 to November 2020 were retrospectively reviewed, and adult patients with the diagnosis of BVFI after intubation were included. Data on comorbidities, duration of intubation, laryngoscopy, and decannulation status was analyzed.

Results Out of the 68 patients included in the present study, $60.3 \%$ were male, and the mean duration of intubation $14.3 \pm 8.5$ days. A total of $94 \%$ of the patients were intubated for at least 7 days, diabetic, and/or obese. Although association with prolonged intubation $>7$ days was not significant $(p=0.064)$, complete BVFI on fiberoptic exam $(n=47)$ was significantly associated with tracheostomy dependence both in the entire cohort $(p=0.036)$ and in the 56 patients with tracheostomy $(p=0.0086)$. Patients without cardiovascular disease (CVD) were less likely to be tracheostomy dependent compared with those with CVD (odds ratio [OR]: 0.23 [0.053-0.79]; $p=0.028$ ).

Conclusions We identified duration of intubation, DM, and obesity as potential risk factors for PGS. Complete immobility and CVD were significantly associated with tracheostomy dependence. Our findings may have important implications for earlier tracheostomy in high-risk intubated patients, as well as for closer monitoring of disease progression and earlier intervention in those predisposed to tracheostomy dependence.
\end{abstract}

received

July 15,2021

accepted after revision

September 19, 2021

published online

February 4, 2022
DOI https://doi.org/ 10.1055/s-0041-1741435. ISSN 1809-9777.

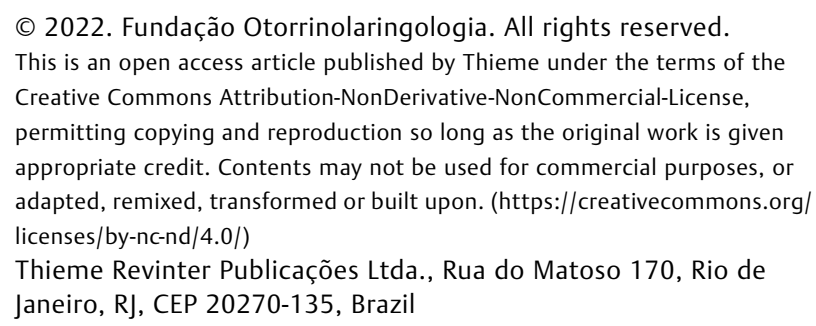

(c) 2022. Fundação Otorrinolaringologia. All rights reserved. This is an open access article published by Thieme under the terms of the Creative Commons Attribution-NonDerivative-NonCommercial-License, permitting copying and reproduction so long as the original work is given appropriate credit. Contents may not be used for commercial purposes, or adapted, remixed, transformed or built upon. (https://creativecommons.org/ licenses/by-nc-nd/4.0/) Thieme Revinter Publicações Ltda., Rua do Matoso 170, Rio de Janeiro, RJ, CEP 20270-135, Brazil 


\section{Introduction}

Iatrogenic bilateral vocal fold immobility (BVFI) can be caused by prolonged or traumatic intubation. Sequelae can be devastating, resulting in dyspnea, stridor, and death due to asphyxiation. While the most common iatrogenic cause of BVFI is neural injury after surgery, such as thyroidectomy, BVFI can result from structural injury. Intubation may lead to the development of BVFI from posterior glottic stenosis (PGS) in adults, secondary to the vulnerability of the posterior glottic mucosa. ${ }^{1,2}$ Weymuller et al. quantified the degree of endotracheal tube (ETT) pressure in canine larynges, demonstrating pressures that are significantly greater than capillary perfusion pressure. These pressures were highest at the posterolateral aspect of the glottis, where injury occurred in the specimens. ${ }^{3}$

The initial site of development of PGS is at the posterior commissure, a thin mucosal layer overlying the laryngeal cartilage. Significant pressure from the ETT is placed in this region, which leads to inflammation, mucosal disruption, ulceration, and the development of granulation and fibrosis at the posterior glottis. Progression of disease is demonstrated by ankylosis of the cricoarytenoid joint and medial fixation of the arytenoid vocal processes, resulting in a significantly narrowed airway with limited vocal fold abduction. ${ }^{1,4-6}$ Bogdasarian et al. established the most commonly used PGS classification (I-IV) based on the progressive fibrosis of the cricoid and arytenoid cartilages. ${ }^{1}$ Advanced stage is more often associated with respiratory symptoms, while voice and swallowing are typically less affected.

Identifying individuals at risk for the development of postintubation PGS may help us better understand how to prevent this condition and/or intervene earlier to manage these patients. Specifically, the association of body mass index (BMI) and diabetes mellitus (DM) with postintubation PGS has not been extensively described, despite the implications on ETT size and wound healing for both of these conditions.

Treatment typically involves procedures to open the airway with the goal of avoiding a tracheostomy or decannulation if a tracheostomy is present. This often comes at a cost to the patient, sacrificing voice quality and increasing aspiration as the airway is opened surgically. Several studies have identified certain factors in patients with laryngotracheal stenosis that are associated with prolonged tracheostomy placement. ${ }^{7-9} \mathrm{To}$ date, only one study has evaluated risk factors solely in patients with a diagnosis of postintubation PGS. ${ }^{5}$ The pathophysiology of this condition is unique, and factors associated with both the development and consequences of PGS after intubation may differ from other etiologies.

In the present article, we review the risk factors associated with PGS development to better understand how to prevent and manage this condition. A secondary aim is to highlight the variables associated with successful decannulation or maintenance of an adequate airway without the need for tracheostomy. We hypothesize that obesity, DM, smoking history, cardiovascular conditions, and prolonged intubation $>7$ days are potential risk factors for the inci- dence of PGS and worsening degree of immobility. We suspect that these same risk factors may have implications on the need for prolonged tracheostomy, in addition to complete immobility with compromised abduction.

\section{Materials and Methods}

\section{Patient Identification}

A review of institutional records at our tertiary care institution was conducted to identify patients with BVFI seen as outpatients by the senior author (Rosow D. W.) at the Department of Otolaryngology of our tertiary care institution between October 2011 and November 2020 using ICD ICD-10 code J38.02. The study was approved by the institutional review board at our tertiary care institution (IRB \# 20210028). Patients $>18$ years old were included in the study if they had a diagnosis of bilateral vocal fold paralysis or of paresis after intubation. Patients were excluded if the etiology of BVFI was unrelated to intubation. Individuals $<18$ years old, pregnant women, radiated patients, and prisoners were excluded.

\section{Data Collection}

Relevant patient details were collected from the electronic medical record system. We reviewed demographic data including age, gender, race, ethnicity, body mass index (BMI), tobacco and alcohol use, and comorbidities including hypertension (HTN), cardiovascular disease (CVD), diabetes mellitus (DM), gastroesophageal reflux disease, and obstructive lung disease. Tobacco and alcohol use were defined as never, former, or current user.

Additional information regarding the hospital stay of the patients was gathered from chart review. We extracted data regarding duration of intubation and ETT size when information was available. Given that the majority of the hospitalizations of the patients was at outside institutions, verbal consent was obtained from the patient prior to request of outside medical records.

Symptomatology was assessed both at initial presentation and at the most recent follow-up. Voice changes were documented as positive if the patient reported subjective changes other than mild hoarseness. Fiberoptic laryngoscopy exam videos were reviewed by the senior author to assess the degree of immobility. "Complete" indicated that the patient had total BVFI; "partial" included either those with unilateral immobility and contralateral hypomobility or those with bilateral hypomobility.

The patients were categorized according to existing tracheostomy status, whereby those with no existing tracheostomy included both those who were decannulated after tracheostomy placement or if tracheostomy was avoided altogether. In addition, decannulation status in patients who had a history of tracheostomy was evaluated separately.

\section{Statistical Analysis}

All statistical analyses were performed by using RStudio statistical software version 4.0.3 (R Foundation for Statistical Computing, Vienna, Austria). Associations between categorical 
variables were analyzed using the chi-squared or the Fisher exact test, as appropriate. Simple logistic regression was performed to explore the relationship between each potential predictor with binomial outcomes, in order to determine which factors may be predictive for complete BVFI, decannulation, and existing tracheostomy as described above. Multivariable analyses were subsequently performed using binomial logistic regression to predict outcomes by adjusting covariates. The variables included in the model were gender, age, race, ethnicity, smoking and alcohol history, CVD, diabetes, BMI, and duration of intubation. The reduced models were selected using backward stepwise selection with the lowest Akaike Information Criteria (AIC) as criteria for best fit model. Statistical significance was defined as p-value $<0.05$, and Bonferonni correction for multivariate analyses yielded pvalue $<0.017$. Power analyses were also performed for each of the outcomes and are reviewed in - Supplemental Table S1 (online only).

\section{Results}

\section{Demographics}

Demographic data for the 68 patients are shown in - Table 1. The mean age was $53.9 \pm 15.3$ years old, with an average follow-up period of 1.80 years. The majority of the patients was male $(60.3 \%)$, and $40 \%$ met criteria for obesity, that is BMI $\geq 30$. More than half of the patients had been smokers at some point $(n=36)$, whereas only 18 patients had a positive alcohol history. A total of 43 patients had HTN or CVD, and 23 had DM. The median duration of intubation was 14 days, with a mean of $14.3 \pm 8.5$ days. Out of the 52 patients with documented length of intubation, 49 (94\%) were intubated for at least 7 days, diabetic, or obese (BMI $\geq 30$ ), or a combination of these factors. Of the remaining 3 patients, 2 were overweight $(\mathrm{BMI} \geq 25$ ) and had documented traumatic intubations.

At the initial clinic visit, 29 patients without tracheostomy had audible stridor, and 37 reported voice changes. Fiftysix patients had tracheostomy either while in the hospital for prolonged intubation or subsequent to intubation secondary to airway concern. The surgical approaches for BVFI were primarily endoscopic $(n=38)$. Findings before and after endoscopic intervention are depicted in -Figure $\mathbf{1}$.

\section{Degree of Immobility}

The degree of immobility was assessed for each patient on fiberoptic laryngoscopy. Forty-eight patients (70.6\%) had complete BVFI. No significant association between degree of immobility and factors including gender, age, race, ethnicity, BMI, HTN/CVD, DM, smoking history, and duration of intubation was identified on bivariate analysis. On multivariate analysis, the effect of duration of intubation did not quite achieve significance (odds ratio [OR]: $0.27 ; 95 \%$ confidence interval $[\mathrm{CI}]$ : 0.067-1.06; $p=0.064$ ) (-Table 2 ).

\section{Existing Tracheostomy}

No existing tracheostomy was noted in 56 patients (82.3\%), whereas 12 (17.6\%) remained with tracheostomy at follow-
Table 1 Patient demographics and other characteristics

\begin{tabular}{|c|c|}
\hline Characteristics & $\begin{array}{l}n(\%) \\
(n=68)\end{array}$ \\
\hline \multicolumn{2}{|l|}{ Age } \\
\hline$<60$ & $48(70.6 \%)$ \\
\hline$\geq 60$ & $20(29.4 \%)$ \\
\hline \multicolumn{2}{|l|}{ Gender } \\
\hline Male & $41(60.3 \%)$ \\
\hline Female & $27(39.7 \%)$ \\
\hline \multicolumn{2}{|l|}{ Race } \\
\hline White & $51(75.0 \%)$ \\
\hline Black & $17(25.0 \%)$ \\
\hline \multicolumn{2}{|l|}{ Ethnicity } \\
\hline Hispanic & $19(27.9 \%)$ \\
\hline Non-hispanic & $49(72.1 \%)$ \\
\hline \multicolumn{2}{|c|}{ Smoking History } \\
\hline Yes & $36(52.9 \%)$ \\
\hline No & $32(47.1 \%)$ \\
\hline \multicolumn{2}{|l|}{ Alcohol Use } \\
\hline Yes & $18(26.5 \%)$ \\
\hline No & $50(73.6 \%)$ \\
\hline \multicolumn{2}{|l|}{ Comorbidities: } \\
\hline \multicolumn{2}{|c|}{ Cardiovascular Disease } \\
\hline Yes & $43(63.2 \%)$ \\
\hline No & $25(36.8 \%)$ \\
\hline \multicolumn{2}{|l|}{ Diabetes } \\
\hline Yes & $23(33.8 \%)$ \\
\hline No & $45(66.2 \%)$ \\
\hline \multicolumn{2}{|l|}{ Asthma/COPD } \\
\hline Yes & $11(16.2 \%)$ \\
\hline No & $57(83.8 \%)$ \\
\hline \multicolumn{2}{|l|}{ Overweight $^{\mathrm{a}}$} \\
\hline Yes & $49(72.1 \%)$ \\
\hline No & $19(27.9 \%)$ \\
\hline \multicolumn{2}{|l|}{ Obese $e^{\mathrm{b}}$} \\
\hline Yes & $28(41.2 \%)$ \\
\hline No & $40(58.8 \%)$ \\
\hline \multicolumn{2}{|c|}{ Intubation Duration } \\
\hline$>7$ days & $38(55.9 \%)$ \\
\hline$\leq 7$ days & $14(20.6 \%)$ \\
\hline Unknown & $16(23.5 \%)$ \\
\hline \multicolumn{2}{|c|}{ Bilateral Immobility } \\
\hline Complete & $48(70.6 \%)$ \\
\hline Partial & $20(29.4 \%)$ \\
\hline \multicolumn{2}{|l|}{ Symptoms: } \\
\hline Voice Changes & \\
\hline
\end{tabular}


Table 1 (Continued)

\begin{tabular}{|l|l|}
\hline Characteristics & $\begin{array}{l}\boldsymbol{n}(\mathbf{( \% )} \\
(\boldsymbol{n}=\mathbf{6 8 )}\end{array}$ \\
\hline Yes & $37(54.4 \%)$ \\
\hline No & $31(45.5 \%)$ \\
\hline Dysphagia & $10(14.7 \%)$ \\
\hline Yes & $58(85.3 \%)$ \\
\hline No & $29(42.6 \%)$ \\
\hline Stridor & $39(57.4 \%)$ \\
\hline Yes & \\
\hline No & $56(82.4 \%)$ \\
\hline Ever Underwent Tracheostomy & $12(17.6 \%)$ \\
\hline Yes & \\
\hline No & $28(41.2 \%)$ \\
\hline Decannulated & $28(41.2 \%)$ \\
\hline Yes & $12(17.6 \%)$ \\
\hline No &
\end{tabular}

${ }^{\mathrm{a} O v e r w e i g h t ~ i n c l u d e s ~ p a t i e n t s ~ w i t h ~} \mathrm{BMI} \geq 25$.

bobese includes patients with $\mathrm{BMI} \geq 30$.

up. Patients with complete BVFI on fiberoptic exam were significantly less likely to have no tracheostomy compared to those with partial BVFI (OR: 0.25 ; 95\%CI: 0.064-0.80; $p=0.028$ ). Multivariate analysis demonstrated a significant effect of HTN/CVD on the presence of a tracheostomy. Specifically, the odds of having an existing tracheostomy in patients without HTN/CVD was 0.23 times that of those with HTN/HLD (OR: $0.23 ; \quad 95 \% C l: 0.053-0.79 ; \quad p=0.028$ ). The degree of immobility was also significantly correlated, as patients with complete BVFI were more likely to have an existing tracheostomy compared with partial BVFI (OR: 4.15; 95\%CI: 1.18-17.70; $p=0.036$ ) (-Table 3).

\section{Status}

Half of the patients who had a tracheostomy placed either as inpatients or after hospitalization due to airway concerns were subsequently decannulated. We found a significant association between complete immobility and failure to be decannulated (OR: 0.19; 95\%CI: 0.047-0.66; $p=0.012$ ). On multivariable analysis including all variables and after adjustment, there was a very significant relationship between the degree of immobility and decannulation status, as patients with complete BVFI were more likely to fail decannulation (OR: 6.23; 95\%CI: 1.73-27.86; $p=0.0086$ ). (-Table 4)

\section{Discussion}

Optimal timing for a tracheostomy in intubated patients is hotly debated. In a large multicenter randomized control trial, Young et al. found no difference in 30-day or 2-year mortality between early ( $\leq 4$ days) and late ( $>10$ days)

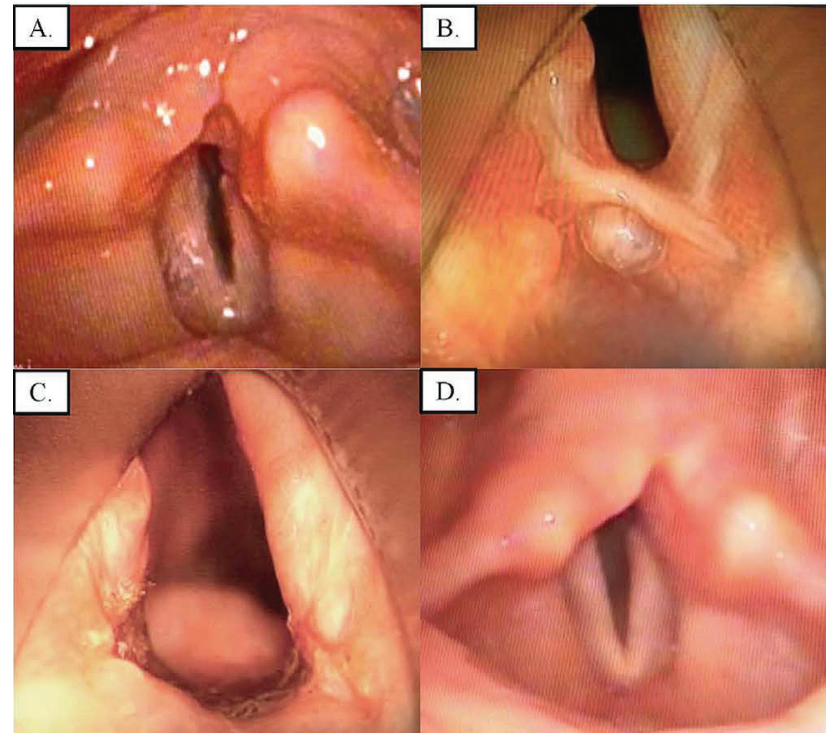

Fig. 1 (A) Videostroboscopy demonstrating posterior commissure ulceration and bilateral vocal fold paralysis in the median position with severely narrowed airway. (B) Thick posterior glottic scar band with fibrosis and cricoarytenoid joint ankylosis on direct laryngoscopy. (C) Appearance of the posterior glottis after laser and balloon dilation of scar band. (D) Normal appearing posterior glottic mucosa and improved airway patency 6 months postoperatively.

tracheostomy. However, this study primarily focused on mortality, and none of the measured secondary outcomes concerned laryngeal injury. ${ }^{10}$ Meanwhile, it is well-known that prolonged intubation has important implications for the development of laryngeal injury, which can occur in up to $20 \%$ of individuals. ${ }^{11}$ The correlation between BVFI and prolonged intubation has been shown previously. ${ }^{5,12,13} \mathrm{~A}$ landmark study published in 1984 demonstrated a $100 \%$ increase in the development of PGS for patients intubated for between 11 and 24 days compared with those intubated for between 5 and 10 days. $^{13}$ Similarly, the mean duration of intubation in our cohort was 14.3 days, with $73 \%$ intubated for $>7$ days.

Several studies have highlighted a relationship between large ETT size and the development of SGS. ${ }^{9,14}$ The implications of larger ETT in PGS has been described in only one study, by Hillel et al., who noted that all male cases of PGS were intubated with a size $8.0 \mathrm{ETT}^{5}$ Male patients may be more prone to the development of PGS secondary to larger ETT size. In our study, the majority of patients were male (60.3\%). Given the retrospective design, $<1 / 4$ of our patients had data regarding ETT size, so we were unable to describe the effect of this variable.

Height has also been implicated as an important factor in ETT selection. One study analyzed tracheal luminal diameters on computed tomography (CT) and concluded a significant relationship between the height of the patient and airway diameter only in males. They deduced that, depending on height in male patients, larger ETT may not always be indicated. ${ }^{15} \mathrm{~A}$ case-control study by Katsantonis et al. evaluated risk factors for postintubation PGS and found that shorter height was significantly predictive of PGS. This 
Table 2 Bivariate and multivariate logistic regression analysis for degree of immobility

\begin{tabular}{|c|c|c|c|c|c|}
\hline \multicolumn{6}{|c|}{ Bivariate Analysis } \\
\hline \multicolumn{2}{|c|}{ Prognostic Factor } & Category (n) & Complete & OR $(95 \% \mathrm{Cl})$ & p-value \\
\hline \multirow{2}{*}{\multicolumn{2}{|c|}{ Gender }} & Male (41) & 31 & Reference (1) & \multirow[t]{2}{*}{0.26} \\
\hline & & Female (27) & 17 & $0.055(0.019-1.59)$ & \\
\hline \multirow{2}{*}{\multicolumn{2}{|c|}{ Age (years old) }} & $<60(48)$ & 35 & Reference (1) & \multirow[t]{2}{*}{0.51} \\
\hline & & $\geq 60(20)$ & 13 & $0.69(0.23-2.18)$ & \\
\hline \multirow{2}{*}{\multicolumn{2}{|c|}{ Race }} & White (51) & 36 & Reference (1) & \multirow[t]{2}{*}{1.00} \\
\hline & & Black (17) & 12 & $1.0(0.31-3.59)$ & \\
\hline \multirow{2}{*}{\multicolumn{2}{|c|}{ Ethnicity }} & Hispanic (19) & 16 & Reference (1) & \multirow[t]{2}{*}{0.15} \\
\hline & & Non-hispanic (49) & 32 & $0.35(0.075-1.25)$ & \\
\hline \multirow{2}{*}{\multicolumn{2}{|c|}{ Smoking history }} & Yes (36) & 26 & Reference (1) & \multirow[t]{2}{*}{0.75} \\
\hline & & No (32) & 22 & $0.85(0.29-2.42)$ & \\
\hline \multirow{2}{*}{\multicolumn{2}{|c|}{ Alcohol history }} & Yes (18) & 13 & Reference (1) & \multirow[t]{2}{*}{1.00} \\
\hline & & No $(50)$ & 35 & $0.87(0.233-2.79)$ & \\
\hline \multirow{2}{*}{\multicolumn{2}{|c|}{ Obesity }} & Yes (18) & 19 & Reference (1) & \multirow[t]{2}{*}{0.68} \\
\hline & & No (40) & 29 & $1.25(0.43-3.60)$ & \\
\hline \multirow{2}{*}{\multicolumn{2}{|c|}{ Diabetes }} & Yes (23) & 17 & Reference (1) & \multirow[t]{2}{*}{0.78} \\
\hline & & No (45) & 31 & $0.78(0.24-2.34)$ & \\
\hline \multirow{2}{*}{\multicolumn{2}{|c|}{ Hypertension/Cardiovascular disease }} & Yes (43) & 29 & Reference (1) & \multirow[t]{2}{*}{0.58} \\
\hline & & No (25) & 19 & $1.53(0.51-4.95)$ & \\
\hline \multirow{2}{*}{\multicolumn{2}{|c|}{ Duration of intubation $^{\text {a }}$}} & $>7$ days $(38)$ & 29 & Reference (1) & \multirow[t]{2}{*}{0.075} \\
\hline & & $\leq 7$ days $(14)$ & 7 & $0.31(0.083-1.12)$ & \\
\hline \multicolumn{6}{|c|}{ Multivariate Analysis } \\
\hline Model & Prognostic factor & Category & & OR $(95 \% \mathrm{Cl})$ & p-value \\
\hline \multirow[t]{2}{*}{1} & \multirow[t]{2}{*}{ Duration of intubation } & $>7$ days & & Reference (1) & \multirow[t]{2}{*}{0.082} \\
\hline & & $\leq 7$ days & & $0.27(0.062-1.16)$ & \\
\hline \multirow[t]{2}{*}{2} & \multirow[t]{2}{*}{ Duration of intubation } & $>7$ days & & Reference (1) & \multirow[t]{2}{*}{0.064} \\
\hline & & $\leq 7$ days & & $0.27(0.067-1.07)$ & \\
\hline
\end{tabular}

Abbreviations: OR $(95 \% \mathrm{Cl})$, estimated odds ratio and corresponding $95 \%$ confidence interval.

In bold $p<0.05$.

Multivariable logistic regression model 1 includes degree of immobility (complete versus partial) plus the following 10 variables: Gender (male versus. female), age ( $<60$ versus $\geq 60$ years old), race (white versus black), ethnicity (Hispanic versus non-Hispanic), smoking history (yes versus no), alcohol history (yes versus no), obesity (yes versus no), diabetes (yes versus no), hypertension/cardiovascular disease (yes versus no), and duration of intubation ( $>7$ versus $\leq 7$ days).

Multivariable logistic regression model 2 includes the particular marker(s) reported plus the following 2 variables: gender, ethnicity, and duration of intubation.

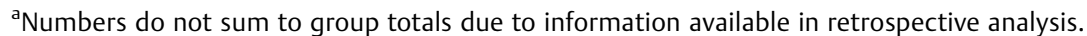

may be secondary to ETT that are too large for shorter patients. ${ }^{16}$ Larger multicenter studies may be important for implementing updated algorithms for ETT selection.

Prior studies have demonstrated a relationship between comorbid conditions and the development of airway pathology after intubation. ${ }^{12,17}$ Diabetic patients are at an eightfold increased risk of development of laryngeal stenosis. Diabetes leads to a systemic hypoperfusion, which leads to poor wound healing and exacerbates ulceration and scarring seen in PGS. ${ }^{4,18}$ Similarly, ischemia in patients with CVD may contribute to PGS via a similar mechanism. Both CVD and DM were noted to be significant risk factors for PGS in the aforementioned study by Hillel et al. In our cohort, over
$1 / 3$ of patients were diabetic, and nearly $2 / 3$ had HTN or ischemia, highlighting that these may be risk factors for the development of PGS.

In contrast to DM and prolonged intubation, the effect of BMI on the development of postintubation BVFI has not been well-described. D'Anza et al. identified a significant inverse correlation between BMI and tracheal size on imaging in patients undergoing tracheostomy. ${ }^{19}$ In our clinical experience, we have seen that typically larger patients with higher BMI are intubated with larger ETT, particularly in the emergency, trauma, and critical care settings. As noted above, this has important implications for the development of BVFI, given that large ETT may predispose to the mucosal 
Table 3 Bivariate and multivariate logistic regression analysis for existing tracheostomy

\begin{tabular}{|c|c|c|c|c|c|}
\hline \multicolumn{6}{|c|}{ Bivariate Analysis } \\
\hline \multicolumn{2}{|c|}{ Prognostic factor } & Category $(N)$ & Yes & OR (95\% Cl) & p-value \\
\hline \multirow{2}{*}{\multicolumn{2}{|c|}{ Gender }} & Male (41) & 16 & Reference (1) & \multirow[t]{2}{*}{0.066} \\
\hline & & Female (27) & 12 & $1.25(0.46-3.36)$ & \\
\hline \multirow{2}{*}{\multicolumn{2}{|c|}{ Age (years old) }} & $<60(48)$ & 22 & Reference (1) & \multirow[t]{2}{*}{0.23} \\
\hline & & $\geq 60(20)$ & 6 & $0.51(0.16-1.49)$ & \\
\hline \multirow{2}{*}{\multicolumn{2}{|c|}{ Race }} & White (51) & 21 & Reference (1) & \\
\hline & & Black (17) & 7 & $1.00(0.32-3.03)$ & 1.00 \\
\hline \multirow{2}{*}{\multicolumn{2}{|c|}{ Ethnicity }} & Hispanic (19) & 11 & Reference (1) & \multirow[t]{2}{*}{0.086} \\
\hline & & Non-Hispanic (49) & 17 & $0.39(0.13-1.13)$ & \\
\hline \multirow{2}{*}{\multicolumn{2}{|c|}{ Smoking history }} & Yes (36) & 15 & Reference (1) & \multirow[t]{2}{*}{0.93} \\
\hline & & No (32) & 13 & $0.96(0.36-2.53)$ & \\
\hline \multirow{2}{*}{\multicolumn{2}{|c|}{ Alcohol history }} & Yes (18) & 6 & Reference (1) & \multirow[t]{2}{*}{0.48} \\
\hline & & No $(50)$ & 22 & $1.50(0.496-4.91)$ & \\
\hline \multirow{2}{*}{\multicolumn{2}{|c|}{ Obesity }} & Yes (18) & 13 & Reference (1) & \multirow[t]{2}{*}{0.46} \\
\hline & & No (40) & 15 & $0.69(0.26-1.85)$ & \\
\hline \multirow{2}{*}{\multicolumn{2}{|c|}{ Diabetes }} & Yes (23) & 11 & Reference (1) & \multirow[t]{2}{*}{0.43} \\
\hline & & No (45) & 17 & $0.66(0.24-1.84)$ & \\
\hline \multirow{2}{*}{\multicolumn{2}{|c|}{ Hypertension/Cardiovascular disease }} & Yes (43) & 20 & Reference (1) & \multirow[t]{2}{*}{0.24} \\
\hline & & No (25) & 8 & $0.54(0.19-1.49)$ & \\
\hline \multirow{2}{*}{\multicolumn{2}{|c|}{ Duration of intubation $^{\mathrm{a}}$}} & $>7$ days $(38)$ & 16 & Reference (1) & \multirow[t]{2}{*}{0.68} \\
\hline & & $\leq 7$ days $(14)$ & 5 & $0.76(0.20-2.66)$ & \\
\hline \multirow{2}{*}{\multicolumn{2}{|c|}{ Degree of immobility }} & Partial (20) & 4 & Reference (1) & \multirow[t]{2}{*}{0.028} \\
\hline & & Complete (48) & 24 & $0.25(0.064-0.80)$ & \\
\hline \multicolumn{6}{|c|}{ Multivariate Analysis } \\
\hline Model & Prognostic factor & Category & & OR $(95 \% \mathrm{Cl})$ & p-value \\
\hline \multirow[t]{6}{*}{1} & \multirow[t]{2}{*}{ Degree of immobility } & Partial & & Reference (1) & \multirow[t]{2}{*}{0.025} \\
\hline & & Complete & & $5.52(1.37-29.04)$ & \\
\hline & Hypertension/Cardiovascular disease & Yes & & Reference (1) & 0.045 \\
\hline & & No & & $0.21(0.041-0.90)$ & \\
\hline & Ethnicity & Hispanic & & Reference (1) & 0.077 \\
\hline & & Non-Hispanic & & $0.28(0.61-1.09)$ & \\
\hline 2 & Degree of immobility & Partial & & Reference (1) & 0.036 \\
\hline & & Complete & & $4.15(1.18-17.70)$ & \\
\hline & Hypertension/Cardiovascular disease & Yes & & Reference (1) & 0.028 \\
\hline & & No & & $0.23(0.053-0.79)$ & \\
\hline & Ethnicity & Hispanic & & Reference (1) & 0.065 \\
\hline & & Non-Hispanic & & $0.30(0.076-1.03)$ & \\
\hline
\end{tabular}

Abbreviations: OR $(95 \% \mathrm{Cl})$, estimated odds ratio and corresponding $95 \%$ confidence interval.

In bold $p<0.05$.

Multivariable logistic regression model 1 includes existing tracheostomy (no versus. yes) plus the following 11 variables: Gender (male versus female), age ( $<60$ versus $\geq 60$ years old), race (white versus black), ethnicity (Hispanic versus non-Hispanic), smoking history (yes versus no), alcohol history (yes versus no), obesity (yes versus no), diabetes (yes versus no), hypertension/cardiovascular disease (yes versus no), duration of intubation ( $>7$ versus $\leq 7$ days), and degree of immobility (partial versus complete).

Multivariable logistic regression model 2 includes the following 4 variables: age, hypertension/cardiovascular disease, ethnicity, and degree of immobility.

${ }^{a}$ Numbers do not sum to group totals due to information available in retrospective analysis. 
Table 4 Bivariate and multivariate logistic regression analysis for decannulation status

\begin{tabular}{|c|c|c|c|c|c|}
\hline \multicolumn{6}{|c|}{ Bivariate analysis } \\
\hline \multicolumn{2}{|c|}{ Prognostic factor } & Category (n) & Not decannulated & OR $(95 \% \mathrm{Cl})$ & $p$-value \\
\hline \multirow{2}{*}{\multicolumn{2}{|c|}{ Gender }} & Male (41) & 16 & Reference (1) & \multirow[t]{2}{*}{0.79} \\
\hline & & Female (27) & 12 & $0.87(0.30-2.49)$ & \\
\hline \multirow{2}{*}{\multicolumn{2}{|c|}{ Age (years old) }} & $<60(48)$ & 22 & Reference (1) & \multirow[t]{2}{*}{0.24} \\
\hline & & $\geq 60(20)$ & 6 & $0.49(0.14-1.58)$ & \\
\hline \multirow{2}{*}{\multicolumn{2}{|c|}{ Race }} & White (51) & 21 & Reference (1) & \multirow[t]{2}{*}{0.76} \\
\hline & & Black (17) & 7 & $0.83(0.25-2.74)$ & \\
\hline \multirow{2}{*}{\multicolumn{2}{|c|}{ Ethnicity }} & Hispanic (19) & 11 & Reference (1) & \multirow[t]{2}{*}{0.082} \\
\hline & & Non-Hispanic (49) & 17 & $0.34(0.091-1.11)$ & \\
\hline \multirow{2}{*}{\multicolumn{2}{|c|}{ Smoking history }} & Yes (36) & 15 & Reference (1) & \multirow[t]{2}{*}{1.00} \\
\hline & & No (32) & 13 & $1.00(0.35-2.88)$ & \\
\hline \multirow{2}{*}{\multicolumn{2}{|c|}{ Alcohol history }} & Yes (18) & 6 & Reference (1) & \multirow[t]{2}{*}{0.75} \\
\hline & & No $(50)$ & 22 & $1.22(0.35-4.37)$ & \\
\hline \multirow{2}{*}{\multicolumn{2}{|c|}{ Obesity }} & Yes (18) & 13 & Reference (1) & \multirow[t]{2}{*}{0.28} \\
\hline & & No $(40)$ & 15 & $0.55(0.18-1.60)$ & \\
\hline \multirow{2}{*}{\multicolumn{2}{|c|}{ Diabetes }} & Yes (23) & 11 & Reference (1) & \multirow[t]{2}{*}{0.78} \\
\hline & & No (45) & 17 & $0.86(0.29-2.54)$ & \\
\hline \multirow{2}{*}{\multicolumn{2}{|c|}{$\begin{array}{l}\text { Hypertension/Cardiovascular } \\
\text { disease }\end{array}$}} & Yes (43) & 20 & Reference (1) & \multirow[t]{2}{*}{0.4} \\
\hline & & No (25) & 8 & $0.62(0.20-1.88)$ & \\
\hline \multirow{2}{*}{\multicolumn{2}{|c|}{ Duration of intubation ${ }^{a}$}} & $>7$ days (38) & 16 & Reference (1) & \multirow[t]{2}{*}{0.71} \\
\hline & & $\leq 7$ days $(14)$ & 5 & $1.33(0.30-6.22)$ & \\
\hline \multirow{2}{*}{\multicolumn{2}{|c|}{ Degree of immobility }} & Partial (20) & 4 & Reference (1) & \multirow[t]{2}{*}{0.012} \\
\hline & & Complete (48) & 24 & $5.20(1.52-21.28)$ & \\
\hline \multicolumn{6}{|c|}{ Multivariate analysis } \\
\hline Model & Prognostic factor & Category & & OR $(95 \% \mathrm{Cl})$ & $p$-value \\
\hline \multirow[t]{2}{*}{1} & \multirow[t]{2}{*}{ Degree of immobility } & Partial & & Reference (1) & \multirow[t]{2}{*}{0.016} \\
\hline & & Complete & & $7.17(1.62-42.32)$ & \\
\hline \multirow[t]{2}{*}{2} & \multirow[t]{2}{*}{ Degree of immobility } & Partial & & Reference (1) & \multirow[t]{2}{*}{0.0086} \\
\hline & & Complete & & $6.23(1.73-27.86)$ & \\
\hline
\end{tabular}

Abbreviations: OR $(95 \% \mathrm{Cl})$, estimated odds ratio and corresponding $95 \%$ confidence interval.

In bold $p<0.05$.

Multivariable logistic regression model 1 includes decannulation status (yes versus no) plus the following 11 variables: Gender (male versus female), age ( $<60$ versus $\geq 60$ years old), race (white versus black), ethnicity (Hispanic versus non-Hispanic), smoking history (yes versus no), alcohol history (yes versus no), obesity (yes versus no), diabetes (yes versus no), hypertension/cardiovascular disease (yes versus no), duration of intubation ( $>7$ versus $\leq 7$ days), and degree of immobility (partial versus complete).

Multivariable logistic regression model 2 includes the particular marker(s) reported plus obesity.

${ }^{a}$ Numbers do not sum to group totals due to information available in retrospective analysis.

disruption and subsequent scarring that develops. In our cohort, $94 \%$ of the patients were intubated for at least 7 days, diabetic, and/or obese (BMI $\geq 30$ ). This suggests that higher BMI may be an important risk factor for the development of PGS.

Cigarette smoke has been shown to reduce capillary blood flow, to promote reactive oxygen species within microvasculature, to increase mucin secretion, and to induce an inflammatory response in the upper and lower airways. ${ }^{20}$ More than $50 \%$ of our patient cohort were smokers, indicat- ing that smoking may exacerbate PGS and have a compounding effect on the fibrosis that ensues. Prior studies have noted a relationship between smoking and tracheostomy dependence in patients with PGS, as well as poorer outcomes after surgical intervention for BVFI. ${ }^{9,21}$ We did not elucidate a difference in tracheostomy status based on smoking history. This may be due to the fact that we described smoking as patients who had ever smoked at some point rather than as the current smoking status, since only five patients identified as current smokers. Further evaluation of the impact of 
continued smoking on the outcomes of postintubation BVFI would be an interesting topic for future study.

According to the Bogdasarian classification, Class IV PGS presents with complete BVFI, whereas classes I-III may have hypomobility of one or both vocal folds. No prior studies have reviewed risk factors for the development or consequences of complete versus partial immobility. We deduced an association between prolonged intubation and complete immobility, although it was not significant $(p=0.064)$. The failure to achieve significance may be due to the retrospective design with absent data on intubation duration in nearly half of the patients. Large studies may help to deduce the implication of prolonged intubation on the degree of immobility.

Successful airway outcomes can be defined by decannulation potential or avoidance of a tracheostomy. Compared with BVFI from other entities, patients with postintubation PGS require more commonly surgical interventions to have a favorable outcome. ${ }^{9}$ A meta-analysis by Kremer et al. reviewed 19 articles to analyze the impact on the type of surgical approach and noted that patients with prior open surgery had an increased likelihood of additional surgery and that those who underwent stent placement were less likely to be decannulated. ${ }^{22}$ The effect of various endoscopic approaches would be an interesting topic for future analysis.

Factors associated with decannulation in patients with laryngotracheal stenosis have been studied. Gelbard et al. found that both patients with iatrogenic etiology, for example, postintubation, and diabetic patients were less likely to be decannulated. ${ }^{17}$ Another study by Snow et al. reported that for those with subglottic or tracheal narrowing, airway firm scarring as opposed to granulation had a greater association with tracheostomy dependence. ${ }^{8}$ Applying this notion to PGS, significant scarring would be more likely to result in a higher Bogdasarian classification and increased likelihood of complete immobility. As Snow et al. described for SGS and tracheal stenosis, we similarly identified a significant relationship on multivariate analysis between complete BVFI and decreased likelihood of decannulation.

While the association between ischemia and development of PGS has been reported, the relationship between this risk factor and patient outcome is not well-established. We determined a significant correlation between CVD and tracheostomy dependence. This again may be due to the hypoperfusion also seen in diabetic patients, leading to poor wound healing and rebound fibrosis after intervention. ${ }^{18}$

A recent study by Meenan et al. reviewed factors associated with the development of laryngeal lesions precluding decannulation in 371 tracheostomy patients. Similar to our study, the authors did not observe an association between age, gender, race, DM, or duration of intubation and the likelihood of tracheostomy dependence. Interestingly, they did elucidate a significant association between BMI $\geq 25$ and the development of laryngeal lesions precluding decannulation. ${ }^{7}$ Although a substantial proportion of our patients were overweight, we did not identify a relationship between higher BMI and tracheostomy dependence. This may be due to our smaller sample size and may be better studied with a larger population.
Several limitations are inherent of the retrospective study design. Although we attempted to gather data regarding ETT size and duration from outside hospitals, this proved to be extremely difficult, and documentation was notably poor. As a single institution study, our sample size was relatively small, precluding more in-depth analysis of certain factors, such as current smoking history, ETT size, and the effect of specific interventions. Another limitation was the subjectivity of the vocal fold impairment grading, as the fiberoptic laryngoscopy videos were only reviewed by the senior author. In addition, several patients had limited follow-up. This may have increased bias toward poorer outcomes, as those with tracheostomy may be more inclined to follow-up for additional procedures. Data on the number and nature of surgical interventions was not included, which may have been a confounding factor when assessing the propensity toward decannulation and/or avoidance of tracheostomy.

In the era of the coronavirus (COVID-19) pandemic, assessment of factors associated with the development and consequences of PGS may prove to be exceedingly important. Secondary to high patient mortality and virus aerosolization, the American Academy of Otolaryngology-Head and Neck Surgery (AAO-HNS) guidelines initially recommended intubation duration of at least 2 weeks prior to tracheostomy in COVID-19 patients. In one study, the average duration of intubation in 20 COVID-19 patients was 21.8 days; all patients demonstrated laryngeal pathology on fiberoptic exam at the outpatient visit, and $15 \%$ had PGS. ${ }^{23}$ Our group has started to review factors associated with laryngeal injury and outcomes in patients who have been intubated for COVID-19.

\section{Conclusions}

In the present study, we reviewed variables associated with outcomes in patients with postintubation BVFI. We identified duration of intubation, DM, and obesity as potential risk factors for the development of PGS. Complete immobility may be related to prolonged intubation and was noted to be significantly associated with tracheostomy dependence. Ischemia also appeared to play a significant role, as patients with HTN/CVD were more likely to be tracheostomy dependent. Larger studies with control groups are needed to evaluate the clinical importance of these variables, particularly during a time when the risk of COVID-19 virus aerosolization has dramatically increased the average duration of intubation.

\section{Acknowledgements}

We would like to acknowledge the American BronchoEsophagological Association for giving us the opportunity to present our findings at the 2021 Combined Otolaryngology Spring Meeting.

\section{References}

1 Bogdasarian RS, Olson NR. Posterior glottic laryngeal stenosis. Otolaryngol Head Neck Surg (1979) 1980;88(06):765-772 
2 Courey MS, Bryant GL Jr, Ossoff RH. Posterior glottic stenosis: a canine model. Ann Otol Rhinol Laryngol 1998;107(10 Pt 1):839-846

3 Weymuller EA Jr, Bishop MJ, Fink BR, Hibbard AW, Spelman FA. Quantification of intralaryngeal pressure exerted by endotracheal tubes. Ann Otol Rhinol Laryngol 1983;92(5 Pt 1):444-447

4 Weymuller EA Jr. Laryngeal injury from prolonged endotracheal intubation. Laryngoscope 1988;98(8 Pt 2, Suppl 45)1-15

5 Hillel AT, Karatayli-Ozgursoy S, Samad I, et al; North American Airway Collaborative (NoAAC) Predictors of Posterior Glottic Stenosis: A Multi-Institutional Case-Control Study. Ann Otol Rhinol Laryngol 2016;125(03):257-263

6 Kashima HK. Bilateral vocal fold motion impairment: pathophysiology and management by transverse cordotomy. Ann Otol Rhinol Laryngol 1991;100(9 Pt 1):717-721

7 Meenan K, Bhatnagar K, Guardiani E. Intubation-Related Laryngeal Pathology Precluding Tracheostomy Decannulation: Incidence and Associated Risk Factors. Ann Otol Rhinol Laryngol 2021;130(09):1078-1084

8 Snow GE, Shaver TB, Teplitzky TB, Guardiani E. Predictors of Tracheostomy Decannulation in Adult Laryngotracheal Stenosis. Otolaryngol Head Neck Surg 2020;•••:194599820978276

9 Gadkaree SK, Gelbard A, Best SR, Akst LM, Brodsky M, Hillel AT. Outcomes in Bilateral Vocal Fold Immobility: A Retrospective Cohort Analysis. Otolaryngol Head Neck Surg 2018;159(06): 194599818800462

10 Young D, Harrison DA, Cuthbertson BH, Rowan KTracMan Collaborators. Effect of early vs late tracheostomy placement on survival in patients receiving mechanical ventilation: the TracMan randomized trial. JAMA 2013;309(20):2121-2129

11 O'Connor HH, Kirby KJ, Terrin N, Hill NS, White AC. Decannulation following tracheostomy for prolonged mechanical ventilation. J Intensive Care Med 2009;24(03):187-194

$12 \mathrm{Wu} \mathrm{X}, \mathrm{Su} \mathrm{ZZ}, \mathrm{Hu} \mathrm{LJ}$, et al. [Analysis of the risk factors causing tracheal stenosis after tracheotomy for mechanical ventilation in
560 patients]. Zhonghua Er Bi Yan Hou Tou Jing Wai Ke Za Zhi 2007;42(11):839-842

13 Whited RE. A prospective study of laryngotracheal sequelae in long-term intubation. Laryngoscope 1984;94(03):367-377

14 Halum SL, Ting JY, Plowman EK, et al. A multi-institutional analysis of tracheotomy complications. Laryngoscope 2012;122 (01):38-45

15 Karmakar A, Pate MB, Solowski NL, Postma GN, Weinberger PM. Tracheal size variability is associated with sex: implications for endotracheal tube selection. Ann Otol Rhinol Laryngol 2015;124 (02):132-136

16 Katsantonis NG, Kabagambe EK, Wootten CT, Ely EW, Francis DO, Gelbard A. Height is an independent risk factor for postintubation laryngeal injury. Laryngoscope 2018;128(12):2811-2814

17 Gelbard A, Francis DO, Sandulache VC, Simmons JC, Donovan DT, Ongkasuwan J. Causes and consequences of adult laryngotracheal stenosis. Laryngoscope 2015;125(05):1137-1143

18 Bishop MJ. Mechanisms of laryngotracheal injury following prolonged tracheal intubation. Chest 1989;96(01):185-186

19 D'Anza B, Knight J, Greene JS. Does body mass index predict tracheal airway size? Laryngoscope 2015;125(05):1093-1097

20 Yang J, Yu HM, Zhou XD, et al. Cigarette smoke induces mucin hypersecretion and inflammatory response through the p66shc adaptor protein-mediated mechanism in human bronchial epithelial cells. Mol Immunol 2016;69:86-98

21 Qazi S, Mau T, Tibbetts KM. Impact of Patient Factors and Management Strategies on Outcomes After Transverse Posterior Cordotomy. Laryngoscope 2020

22 Kremer C, Jiang R, Singh A, Sukys J, Brackett A, Kohli N. Factors Affecting Posterior Glottic Stenosis Surgery Outcomes: Systematic Review and Meta-analysis. Ann Otol Rhinol Laryngol 2021;130 (10):1156-1163

23 Naunheim MR, Zhou AS, Puka E, et al. Laryngeal complications of COVID-19. Laryngoscope Investig Otolaryngol 2020;5(06): $1117-1124$ 